\title{
Método Híbrido de Recomendación Adaptativa de Objetos de Aprendizaje basado en Perfiles de Usuario
}

\author{
Paula A. Rodríguez ${ }^{(1)}$, Néstor D. Duque ${ }^{(2)}$ y Demetrio A. Ovalle ${ }^{(1)}$ \\ (1) Universidad Nacional de Colombia, Facultad de Minas, Departamento de Ciencias de la Computación y \\ de la Decisión, Grupo de investigación GIDIA, Medellín-Colombia (e-mail: parodriguezma@unal.edu.co, \\ dovalle@unal.edu.co) \\ (2) Universidad Nacional de Colombia, Departamento de Informática y Computación, Grupo de \\ Investigación GAIA, Manizales-Colombia (e-mail: ndduqueme@unal.edu.co)
}

Recibido Dic. 4, 2015; Aceptado Feb. 4, 2016; Versión final Abr. 1, 2016, Publicado Ago. 2016

\begin{abstract}
Resumen
El objetivo de este artículo es proponer un método híbrido de recomendación adaptativa de objetos de aprendizaje, el cual debe inicialmente realizar la selección de características del perfil del estudiante que mejor lo definan. Los sistemas de recomendación híbrida de materiales educativos digitales apoyan procesos de aprendizaje virtual y ayudan a los estudiantes a encontrar recursos relevantes que se adapten a sus necesidades y preferencias. Se seleccionan los algoritmos de recomendación que se van a aplicar, seguido de la elección en la combinación de los resultados de esas técnicas. Finalmente, se evalúa la integración de técnicas teniendo en cuenta la relevancia que se obtiene de las recomendaciones. Se puede concluir que al utilizar sistemas adaptativos de recomendación híbrida se mejoran los resultados, especialmente cuando la combinación incluye la técnica de filtrado por conocimiento.
\end{abstract}

Palabras clave: recomendación adaptativa; método híbrido de recomendación; objetos de aprendizaje; perfiles de usuario

\section{Hybrid Method for Adaptive Recommendation of Learning Objects based on User Profiles}

\begin{abstract}
The aim of this paper is to propose a hybrid method for adaptive recommendation of learning objects that initially performs a selection of characteristics from the student profile that best define it. Hybrid recommendation systems of digital educational materials support virtual learning processes and help students finding relevant resources that are adapted to their needs and preferences. Then, the recommendation algorithms are selected and applied, followed by the choice of how to combine the results of these techniques. Finally, the integration of techniques is evaluated considering the relevance obtained by applying the recommendation techniques. It can be concluded that using adaptive hybrid recommendation systems, improves the generation of relevant results, especially when the combination should include the knowledge-based filtering technique.
\end{abstract}

Keywords: adaptive recommendation; hybrid recommendation method; learning objects; user profiles 


\section{INTRODUCCIÓN}

Los Sistemas de Recomendación (SR) tienen como principal objetivo entregar a los usuarios resultados de búsquedas de información cercanos a sus preferencias y necesidades, a partir del hecho que a un usuario le guste o no cierto ítem, objeto, medio o información al que podría acceder (Chesani, 2002). Existen muchas técnicas de SR, cada una puede ser usada para diferentes enfoques y en diferentes contextos, de acuerdo a las necesidades (Hdioud et al., 2012), además necesitan gran cantidad de información sobre los usuarios y recursos a recomendar para entregar resultados de calidad. Esto se logra con la caracterización de los estudiantes y la retroalimentación que se realice de las recomendaciones (Li, 2010). Un Sistema de recomendación híbrido es aquel que combina diferentes SR para producir una salida con el objetivo de completar sus mejores características y hacer mejores recomendaciones (Burke, 2007). Estos sistemas de recomendación híbrida pueden dividirse en dos grupos, los de combinación lineal, que son aquellos que crean una lista de recomendaciones sin combinarlas para crear una predicción combinada. Y los de combinación secuencial, donde la salida de una técnica de recomendación es la entrada a otra técnica. En este artículo solo se tuvo en cuenta la combinación lineal, donde cada sistema de recomendación genera sus propios resultados y a través de alguna técnica se combinan para dar mejores recomendaciones.

Para que un SR entregue resultados adaptados necesitan de perfiles que almacenen la información y las preferencias de cada usuario (Cazella, 2010). Según (D’Agostino et al., 2005) un "perfil es el modelado de un objeto en forma compacta mediante sus características primordiales. En el caso de un perfil de usuario de un sistema de software, éste puede comprender tanto datos personales y características del sistema computacional, como también patrones de comportamiento, intereses personales y preferencias. Este modelo de usuario está representado por una estructura de datos adecuada para su análisis, recuperación y utilización".

Realizar herramientas como apoyo al proceso enseñanza-aprendizaje, ha sido el reto a lo largo del tiempo en la educación mundial constantemente en la búsqueda de métodos para propiciar la participación activa del estudiante en el proceso (Tovar et al., 2014). Se quieren entonces entregar materiales educativos conocidos como Objetos de Aprendizaje (OA) que según el IEEE (Institute of Electrical and Electronics Engineers) en (Learning Technology Standards Committee, 2002), pueden considerarse como una entidad digital con características de diseño instruccional, que puede ser usado, reutilizado o referenciado durante el aprendizaje soportado en computador; con el objetivo de generar conocimientos, habilidades, actitudes y competencias en función de las necesidades del alumno. Los OA están almacenados en bibliotecas digitales especializadas llamadas Repositorios de Objetos de Aprendizaje (ROA) donde se alojan múltiples tipos de recursos educativos y sus metadatos. Los ROA se unen para facilitar su administración a través de Federaciones de Repositorios permitiendo el acceso a los contenidos de los OA disponibles en un grupo de repositorios. Una federación ofrece un enfoque unificado de representación de estos repositorios (Acevedo et al., 2010; Tabares et al., 2015).

El objetivo de este artículo es proponer un método híbrido de recomendación adaptativa de OA, utilizando diferentes técnicas existentes en la literatura, se presentan los pasos necesarios para la construcción de un sistema híbrido, se utiliza un modelo de recomendación híbrida donde se combinan las técnicas contenido, colaborativa y conocimiento. El método propone adicionalmente realizar combinaciones de resultados de las técnicas con el fin de entregar OA adaptados al perfil de un aprendiz específico que sean relevantes a su proceso de aprendizaje. Se realizan diferentes combinaciones de tres técnicas de recomendación (Contenido, colaborativo y conocimiento) y se utilizan las medidas de precisión, recall y f1 score para evaluar su desempeño.

\section{TRABAJOS RELACIONADOS}

Al realizar una búsqueda de trabajos relacionados con los SR híbridos aplicados a materiales de aprendizaje basados en un perfiles de usuarios se encontró el trabajo presentado en (Salehi et al., 2013), donde utilizan algoritmos genéticos y realizan dos procesos de recomendación, el primero de ellos trata de las características explícitas representado en una matriz de preferencias del estudiante. La segunda recomendación es con pesos implícitos a los recursos educativos que son considerados como cromosomas en el algoritmo genético para optimizarlos según los valores históricos. En el trabajo de (Zapata et al., 2011), se entregan materiales educativos ajustados al perfil, combinando varios tipos de filtrado, tratando de utilizar toda la información que se tenga disponible sobre objetos y usuarios. Inicialmente, el método preselecciona OA del repositorio, usando una búsqueda con base en los metadatos, luego esos OA pasan por los otros procesos de filtrado para obtener una lista final que será la que mejor se adapte al usuario En este trabajo se combinan varios criterios de filtrado: basado en el contenido, en la actividad colaborativa y en la demografía. 
Por otro lado, hay trabajos donde realizan la revisión de algunos SR híbrida como el de (Burke, 2002) y el de (Vekariya y Kulkarni, 2012), donde señalan las ventajas, desventajas y usos de las técnicas de hacer híbrida la recomendación. En este último trabajo, utilizaron el filtrado colaborativo (medido con el coeficiente de Pearson) y el filtrado basado en contenido, para hacer la validación. Concluyen que el filtrado híbrido compuesto por el filtrado colaborativo y el filtrado basado en contenido, mejora las predicciones del SR. Por último en el trabajo de (Degemmis et al., 2007) proponen un modelo de recomendación hibrida basado en el perfil de usuario donde también realizan la integración de los SR basados en contenido y colaborativo obteniendo buenos resultados.

Con respecto a la determinación de los estilos de aprendizaje de los estudiantes para entregar materiales adaptados a cada perfil específico se puede encontrar trabajos como el de (Puello et al., 2014), donde implementa el test de Felder y Silverman utilizando la plataforma Moodle, su principal objetivo fue determinar el estilo de aprendizaje predominante en un curso específico.

\section{TÉCNICAS DE RECOMENDACIÓN}

Antes de definir el método propuesto es importante señalar que la integración de técnicas de recomendación incluye las siguientes: por contenido (i.e. basada en el perfil de usuario), filtrado colaborativo (i.e. basada en perfiles de usuario similares) y basada en conocimiento (i.e. basada en el historial de navegación). Estas técnicas se combinan a través del modelo que se presenta en la figura 1, el cual se compone de seis módulos, donde se recuperan los OA desde repositorios y federaciones, usando los metadatos descriptivos almacenados de estos objetos. Los perfiles de los estudiantes también están disponibles y se componen de sus datos personales, preferencias y estilo de aprendizaje. Por lo tanto, hay tres módulos de recomendación uno para cada una de las técnicas seleccionadas; un cuarto módulo que realiza el proceso de integración utilizando los resultados de las recomendaciones y finalmente se tienen dos módulos que manejan la información sobre los perfiles, estos perfiles y están modelados en OWL (Ontology Web Language), lenguaje de la WEB para representación de ontologías de dominio y los metadatos de los OA, que están almacenados en la Federación de Repositorios de Objetos de Aprendizaje Colombia (FROAC).



Fig. 1. Modelo híbrido de técnicas de recomendación que apoya el método propuesto

\section{MÉTODO PROPUESTO}

El método que se propone está compuesto por 4 etapas (ver figura 2) para construir SR híbridos, los pasos que deben seguirse se enumeran a continuación:

1. Seleccionar las características del usuario sobre las que se realizaran las recomendaciones.

2. Seleccionar los SR a utilizar, deben ser mínimo dos para tener un sistema híbrido, implementar las técnicas elegidas.

3. Seleccionar alguna de las técnicas de combinación de sistemas de recomendación e implementarla.

4. Evaluar el desempeño de las recomendaciones utilizando algunas métricas de recuperación de información

A continuación se describen cada una de las etapas.

\section{Etapa 1: Identificación de Características del Usuario}

La primera etapa consiste en la identificación y selección de las características del perfil de usuario que describen al estudiante en su rol de usuario en el SR. Entre las características que se incluyen, están los datos personales, tales como su identificación, nombre, sexo, lugar de origen, nivel de estudios, carrera, institución donde estudia. También se deben incluir las características del contexto del usuario y otras referentes a su historial académico incluyendo los logros o competencias obtenidas y las actividades educativas desarrolladas con los recursos utilizados. 


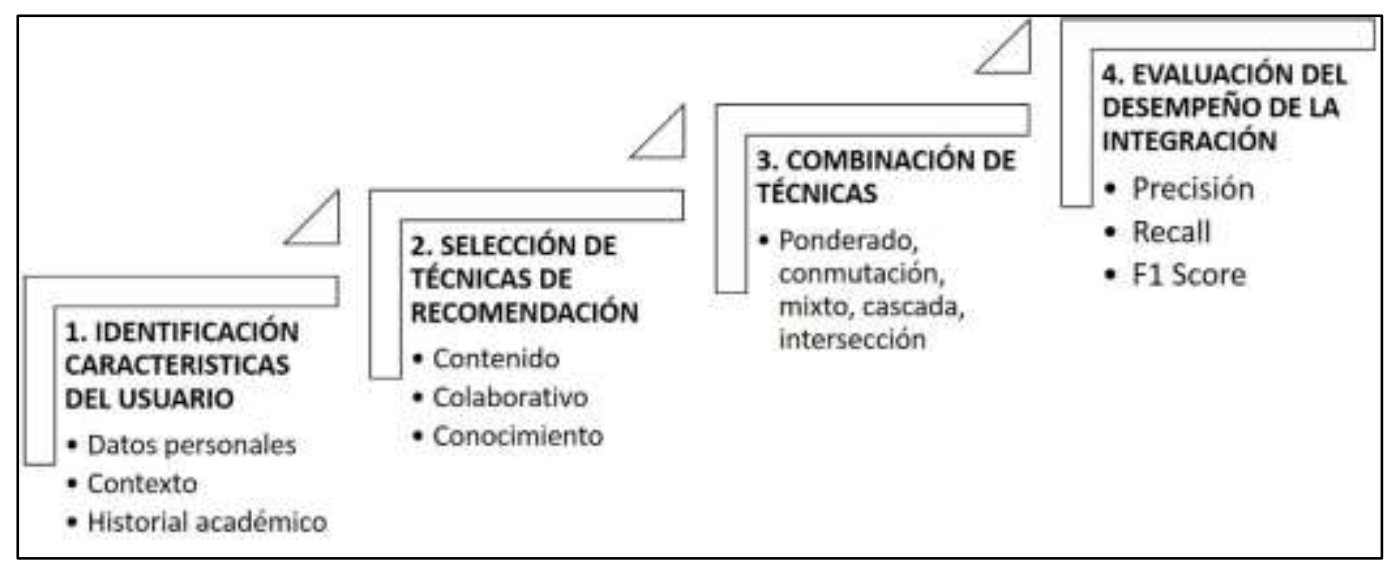

Fig. 2. Método propuesto para la construcción de SR híbridos

Adicionalmente, algunos estudios afirman que el estilo de aprendizaje es la característica más relevante para entregar materiales educativos adaptados (Alonso et al., 1997) (Klašnja-Milićević et al., 2011). El estilo de aprendizaje se refiere a las estrategias para la recopilación de nueva información y adaptación de la misma a su entorno. Existen diferentes modelos para representar el estilo de aprendizaje de un estudiante. En (González et al., 2009) se presenta la combinación de los modelos de estilos de aprendizaje VARK y FSLSM obteniendo buenos resultados para caracterizar el perfil del estudiante y de esta manera entregar al estudiante los materiales educativos adaptados. El modelo de estilos de aprendizaje de VARK propuesto por Neil Fleming y Colleen Mills es un instrumento para determinar la preferencia de los estudiantes al procesar la información desde el punto de vista sensorial. De esta forma las modalidades sensoriales que identificaron son las siguientes: Visual, Auditivo, Lecto-escritor y Kinestésico. Por otro lado el modelo de FSLSM está diseñado a partir de cuatro escalas bipolares, que son Activo-Reflexivo, Sensorial-Intuitivo, Visual-Verbal y Secuencial-Global. Con base en estas escalas, Felder y Silverman han descrito la relación de los estilos de aprendizaje con las preferencias de los estudiantes vinculando los elementos de motivación en el rendimiento escolar (González et al., 2009)

\section{Etapa 2: Selección de Técnicas de Recomendación}

Existen muchas técnicas de recomendación, cada una usada en enfoques y contextos diferente y que requieren información diversa tanto del usuario como del material a recomendar (Hdioud et al., 2012). Así, para el caso de materiales educativos los SR debería ser capaz de recomendar recursos adaptados a un estudiante específico. Burke clasifica los SR en (Burke, 2002):

i) SR basados en el Contenido: realizan la recomendación con base a un perfil de usuario creado que almacena sus intereses, el proceso de recomendación se hace sobre las características presentes en los ítems.

ii) SR Colaborativos: construyen la recomendación como una agregación estadística/probabilística de las preferencias de otros usuarios.

iii) SR basados en Conocimiento: hace recomendaciones según el historial de navegación de un usuario, este historial está almacenado con el fin de obtener las preferencias e intereses del usuario.

El enfoque híbrido, busca la combinación de varias técnicas de recomendación con el objetivo de completar sus mejores características y hacer mejores recomendaciones (Cazella et al., 2010; Burke, 2002). Aunque los SR híbridos tienen grandes ventajas como entregar recomendaciones de alta calidad, su presencia en la Web es baja, debido a su complejo desarrollo ya que se debe tener en cuenta la gestión de un modelo de usuario, tener mecanismos para definir y clasificar la información que puede ser recomendada, la definición de técnicas adaptativas, además de la definición de correspondencias entre los usuarios para seguir con las estrategias de adaptación. Y finalmente, se vuelve un desafío la tarea de seleccionar la mejor técnica de combinación para presentar los resultados.

\section{Etapa 3: Combinación de Técnicas}

Para realizar la combinación de técnicas se deben aplicar al menos dos de ellas, Burke propone diferentes métodos de combinación que se explican en esta sección (Burke, 2002). 
i) Método ponderado: Para cada resultado de cada técnica de recomendación se le da un puntaje y a través de un consenso se combinan los resultados. Donde se combinan las puntuaciones o votos para producir una única recomendación. Esta combinación puede ser aplicada utilizando una función que pondera la importancia de cada uno de los sistemas de recomendación. El problema de este método es que el valor relativo a las diferentes técnicas es uniforme, esto no siempre es verdadero porque depende de la cantidad de información (evaluaciones, usuarios similares, entre otros) con la que se cuenta. La ponderación también puede ser definida en función a una retroalimentación (puntuación) que dan los usuarios a las recomendaciones.

ii) Método de Conmutación: El sistema conmuta entre las técnicas de recomendación en función de la situación actual según algún criterio de conmutación analizando los resultados obtenidos en evidencia pasada. Se aplica una de las técnicas, si esta no entrega resultados, se prosigue a aplicar la siguiente técnica. La desventaja de esta técnica es que si al aplicar la primera técnica si se encuentran resultados, las otras técnicas no se ejecutaran y es posible que se queden sin mostrar algunos resultados relevantes para el usuario.

iii) Método Mixto: Se presentan las recomendaciones de diferentes sistemas de recomendación al mismo tiempo. Se ejecutan todas las técnicas de recomendación disponibles y se presentan todos los resultados, su principal ventaja es que puede mostrar nuevos elementos. Su principal desventaja es que se pueden mostrar resultados que no son tan importantes para el usuario.

iv) Método de cascada: Es un proceso por etapas en la cual, una técnica de recomendación se utiliza por primera vez para producir una clasificación gruesa de los ítems candidatos y una segunda técnica refina la recomendación de entre el conjunto de candidatos. Cada una de las recomendaciones refina las recomendaciones dadas por los otros.

v) Método de combinación de características: Se toman las características, de diferentes fuentes de datos, de una técnica de recomendación se procesan y esas características son la entrada a la otra técnica, este método reduce la sensibilidad del sistema al número de usuarios que han valorado un ítem. En este método se combinan las características de los datos en bruto de diferentes fuentes.

vi) Función de aumento: Una característica de salida de una técnica, se usa como una característica de entrada a otra, es decir se utiliza un modelo para generar rasgos para la entrada de un segundo algoritmo.

Meta-nivel: El modelo aprendido por un sistema de recomendación se utiliza como entrada a otro, es decir todo el modelo es la entrada para el segundo algoritmo. Por ejemplo se hace una primera recomendación sobre la base de interés para la comunidad en conjunto y luego se distribuye a los usuarios particulares. Estos modelos, esencialmente manejan vectores de términos y pesos que pueden ser comparados a través de los usuarios para hacer las predicciones.

vii) Intersección de resultados: A pesar que no está en la revisión bibliográfica como una técnica de combinación, desde el punto de vista de la teoría de conjuntos es posible utilizar la operación intersección que permite obtener los elementos comunes resultados de la aplicación de las técnicas de recomendación. Su principal ventaja es que los resultados entregados son los elementos comunes al aplicar los diferentes $\mathrm{SR}$, además se pueden entregar resultados intermedios, intersecciones de solo dos SR.

\section{Etapa 4: Evaluación del Desempeño de la Integración}

Después de tener los resultados de la integración de los SR, se debe evaluar el desempeño, para ello en esta etapa se proponen tres métricas para calcular la calidad de las recomendaciones, usadas comúnmente para evaluar la recuperación de la información. Burke afirma que las medidas de precisión y recall ayudan a evaluar los resultados y definir si se entregaron buenas recomendaciones, donde se analiza la calidad de la recuperación (Burke, 2002; Baeza y Ribeiro, 2011; Cardona y Sánchez, 2010). Cabe señalar que la relevancia es entendida como la importancia que tiene un material educativo (en este caso OA), para apoyar el proceso de aprendizaje de un estudiante específico, adaptándose a sus preferencias y necesidades. Este valor lo determina un grupo de estudiantes que calificaron dicho valor, después de utilizar el OA.

Las tres métricas consideradas son las siguientes:

i) Precisión: esta medida permite conocer el índice del total de materiales recuperados cuántos fueron acertados, es decir cuáles de ellos fueron considerados relevantes. Este índice entre más cercano a 1 es mejor la técnica de recomendación (Ver fórmula 1). 
precision $=\frac{\text { aciertos }}{\text { recuperados }}$

ii) Recall: para utilizar esta medida se debe conocer del conjunto de posibles resultados cuántos son relevantes para el conjunto de estudiantes, y mide cuantos aciertos del SR están dentro del conjunto de relevantes. Este índice entre más cercano a 1 es mejor la técnica de recomendación (Ver fórmula 2).

recall $=\frac{\text { aciertos }}{\text { relevantes }}$

iii) F1 Score: Es la media armónica ponderada de precisión y recall, donde tiende a 1 cuando el numerador es igual al denominador, indicando una mejor técnica de recomendación (Ver fórmula 3).

f1 score $=\frac{2 * \text { precision } * \text { recall }}{\text { precision }+ \text { recall }}$

\section{EXPERIMENTACIÓN Y APLICACIÓN DEL MÉTODO PROPUESTO}

Para evaluar el método propuesto se utilizó un caso de estudio específico teniendo en cuenta un tipo de estudiante con el fin de evaluar el desempeño al combinar los resultados obtenidos de las técnicas de recomendación seleccionadas.

\section{Etapa 1. Identificación de Características del Usuario}

En la identificación de las características del perfil del estudiante se tomó un trabajo previo de los autores (Rodríguez et al., 2013), donde se seleccionaron las siguientes características: (i) Datos personales: Identificación, Nombres, Apellidos, Fecha de Nacimiento, Email, Sexo, Idioma, Usuario, Contraseña, País, Departamento, Ciudad, Dirección, Teléfono; (ii) Preferencias: Idioma, Temas, Formatos; (iii) Estilo de Aprendizaje: utilizando la combinación de los modelos VARK y FSLSM, utilizado en trabajos previos como en la tesis de maestría (Rodríguez et al., 2013); y (iv) Historial de Uso: Identificación de cada OA evaluado, Calificación, Fecha de utilización. En el caso de estudio, los estudiantes seleccionados tienen el estilo de aprendizaje tipo 7: Visual-Global y sus preferencias son la programación, análisis y diseño de algoritmos. Además estos estudiantes evaluaron la relevancia de algunos OA referentes a la programación exhaustiva (evaluación $\geq 4$, en un rango de 1 a 5 , donde 5 representa que el material es importante para su aprendizaje y 1 el material no es importante para él).

\section{Etapa 2. Selección de Técnicas de Recomendación}

En esta etapa se realizaron búsquedas en FROAC (Federación de Repositorios de Objetos de Aprendizaje Colombia (Tabares et al., 2015), que cuenta actualmente con 621 Objetos de aprendizaje) con diferentes palabras, como algoritmos, programación, entre otras, en promedio los resultados fueron de 20 Objetos de Aprendizaje. Encontrando los resultados de la búsqueda se pusieron a consideración del grupo de estudiantes seleccionado y donde debían calificar cuáles eran relevantes para ellos, considerando la relevancia como la importancia que tiene el objeto para apoyar el aprendizaje del tema que se está buscando. Para evitar el sesgo en las técnicas de recomendación a cada estudiante se le puso a evaluar todos los materiales. Después de obtener los resultados de la búsqueda se ejecutaron las técnicas de recomendación propuestas en la tesis de maestría: (Rodríguez-Marín et al., 2013; Ovalle et al., 2014), que se explican a continuación:

Recomendación por contenido: Esta recomendación está basada en el perfil del estudiante, donde se asocian los metadatos de los OA: LearningResourceType, InteractivityLevel, IntendedEndUser Role, Context, Description y Language, con las características del perfil del estudiante como el estilo de aprendizaje, seleccionando aquellos OA que personalicen los resultados.

Recomendación colaborativa: La recomendación basada en filtrado colaborativo, pretende entregar OA a los estudiantes, buscando OA que le gustaron o interesaron a usuarios similares. Siendo la similaridad la representación numérica del grado de semejanza entre dos usuarios, basada en las preferencias sobre ítems comunes. Para hacer este proceso primero se busca un perfil similar, para este caso se seleccionó la distancia del coseno según el estudio presentado en (Rodríguez et al., 2014), enfocándose en 6 características del perfil del estudiante para encontrar la similitud de los usuarios, estos son: nivel de escolaridad, estilo de aprendizaje, nivel educativo, preferencia de idioma, preferencias de tema y preferencia de formato. Recomienda $O A$ del usuario similar que fueron evaluados positivamente y que fueron recientemente utilizados. 
Recomendación basada en conocimiento: Finalmente la recomendación basada en conocimiento, hace recomendaciones buscando OA similares a los que el usuario evaluó positivamente en el pasado. Para esto primero se realiza un proceso de filtrado de los OA evaluados con una calificación igual o superior a 4 y se buscan OA similares, realizando búsquedas por los metadatos título, descripción y palabras clave de cada $\mathrm{OA}$ a recomendar con los $\mathrm{OA}$ evaluados; la medida de similitud utilizada es overlap.

\section{Etapa 3. Combinación de Técnicas}

Al tener los OA resultantes de cada una de las técnicas de recomendación y tener el conjunto de OA relevantes, para cada una de las palabras de búsqueda; se continúa con la etapa 3 del método propuesto, en donde se realiza la combinación de técnicas que se explica a continuación. Para ello se utiliza una notación para referirnos a las tres técnicas de recomendación aplicadas así: A. Recomendación por contenido, B. Recomendación colaborativo, C. Recomendación por conocimiento. De esta forma se realizaron las siguientes combinaciones de técnicas: $A B, B A, A C, C A, B C, C B, A B C, A C B, B A C, B C A, C A B$, CBA. Es importante señalar que para realizar los experimentos propuestos en este artículo no se pueden aplicar las técnicas: Método de combinación de características, función de aumento y meta-nivel, debido que para este artículo solo se cuenta con los resultados de las técnicas, no se hace la recomendación como tal y estas técnicas necesitan combinar las entradas o los modelos de las entradas de los SR para generar los resultados.

Para el método ponderado, se debe contar con un factor de ponderación de cada técnica de recomendación para seleccionar cada uno de los OA resultado. Estos pesos son tentativos y están asociados a las condiciones específicas del sistema sobre el que se realiza la evaluación, pues las técnicas que requieren de datos históricos para poder hacer la recomendación se verá afectado favorablemente ante un volumen alto de registros y en el otro caso su peso debería ser menor. Al no contar con la cantidad de registros evaluados y como queremos evaluar cual técnica puede ser más apropiada, el orden para la combinación será cambiado mostrando todas las posibilidades, el factor de ponderación debe sumar 1. Para este caso de estudio y con el fin de ser uniforme en el proceso, se optó cuando se combinan dos técnicas los factores de ponderación son 0,7 para la primera y 0,3 para la segunda; por el contrario cuando se combinaron las tres técnicas, los factores fueron de 0,5, 0,3 y 0,2, también según el orden de la combinación y proporcional. El peso de cada OA es 1, debido que tienen el mismo valor por ser resultado de la recomendación y en estas técnicas no se tiene en cuenta el orden en que se entregan los resultados. Para conocer cuáles son los OA resultados de este método híbrido, se seleccionan los OA que estén por encima del umbral de 0,6, debido que es la mitad más 1 del factor de ponderación.

Para el método de conmutación se debe contar con un criterio de selección de la técnica de recomendación, para este caso la primera técnica de cada una de las combinaciones es la seleccionada y los resultados son los que generó esa técnica. En el método mixto se muestran todos los resultados de las técnicas combinadas reuniendo los resultados, primero se muestran los de una técnica, luego los de otra y así presenta todos los $\mathrm{OA}$, sin embargo, como estas técnicas no entregan materiales ordenados, no se tiene en cuenta cuál técnica entrega primero los resultados. Con el método Cascada, se aplican filtros a los resultados de las recomendaciones, entonces para el experimento se seleccionó la primera técnica como la que inicia el proceso y a esos resultados se le aplican los del segundo.

Finalmente, con la técnica de intersección de conjuntos, los resultados son los OA comunes a las técnicas que se están combinando, teniendo en cuenta resultados intermedios de las intersecciones cuando se combinan los resultados de los tres SR.

\section{Etapa 4. Evaluación del Desempeño de la Integración.}

Al tener el resultado de cada una de las combinaciones de las diferentes técnicas y conociendo previamente cuales fueron los OA relevantes para el conjunto de estudiantes que se está trabajando, se aplicó la medida de precisión y recall para cada combinación y palabra, después se hizo un promedio con ellas, para entregar resultados consolidados; finalmente se calculó la medida de f1 score. A continuación se presenta la interpretación de los resultados obtenidos.

i) Métrica de precisión: De los OA recuperados por cada una de las combinaciones, se seleccionaron cuántos de ellos fueron relevantes y así se calculó esta métrica por cada palabra, después se hizo un promedio y los resultados se muestran en la figura 3. La precisión es más cercana a 1 cuando todos los elementos recuperados son relevantes. En el eje x están las diferentes técnicas de combinación y en el eje y, está la precisión que va en escala de a 2, desde el 0 al 1. Debido que la precisión varia en ese rango. 




Fig. 3. Resultados de la métrica de precisión

Con los resultados obtenidos se puede concluir para cada técnica de combinación que: (i) el método ponderado presenta buenos resultados al combinar las técnicas conocimiento con contenido y con colaborativo, dando más peso de ponderación a la técnica de conocimiento. Esto debido a la naturaleza de los datos y que se cuenta con evaluaciones previas, que es el insumo para el SR basado en conocimiento; (ii) el método de conmutación presenta mejores resultados cuando se entregan los OA del sistema de recomendación basado en conocimiento; (iii) el método mixto entrega resultados con una precisión no muy alta para todas las combinaciones, esto se debe a que muestra todos los resultados de las técnicas y no todos son relevantes para los estudiantes; y (iv) los métodos de cascada e intersección tienen los mismos resultados cuando se combinan dos técnicas, entregando muy buenos resultados la combinación de técnicas con contenido, esto debido que muchos de los OA resultados recuperados son relevantes. Y son diferentes cuando se combinan las tres técnicas, dando un valor mayor de precisión el método de cascada, debido que se recuperan pocos elementos y de ellos son relevantes. En cambio la técnica de intersección de resultados tiene un mayor número de elementos recuperados.

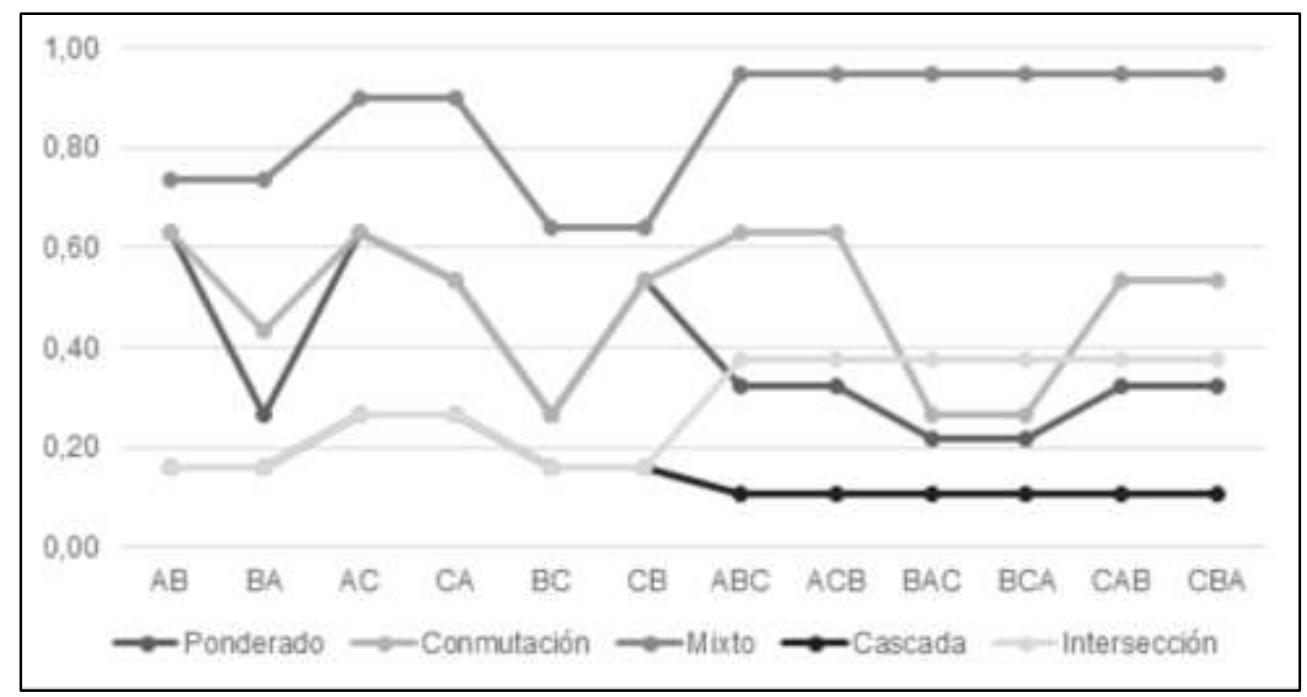

Fig. 4. Resultados de la métrica recall

ii) Métrica de recall: Es más cercano a 1 cuando todos los elementos que son relevantes fueron entregados en la recuperación. Se pueden ver en la figura 4 los resultados para cada combinación.

Con los resultados de esta medida se puede concluir que la técnica de combinación mixta, entrega más cantidad de elementos relevantes, seguida por la técnica de conmutación y ponderado. Adicionalmente, cabe aclarar que la técnica de intersección de resultados, teniendo en cuenta resultados intermedios también entrega un buen índice de recall. Por otro lado la técnica de cascada, no tiene un buen índice de recall, debido a que hace un filtro, donde entrega al final muy pocos resultados, por ello los OA relevantes recuperados son pocos también. 
iii) Métrica f1 score: Esta media permite visualizar los resultados de precisión y recall en una misma métrica (figura 5). Lo que hace esta métrica es que busca la intersección de las métricas precisión y recall y lo divide por la unión de las mismas. El método de combinación mixto entrega un buen resultado, debido que su medida de recall es muy alta.

Por esta razón se consideró importante conocer en promedio la cantidad de elementos que entrega cada técnica de combinación. Para ellos se seleccionaron cuántos objetos fueron recuperados por cada técnica de combinación y cuántos de ellos fueron relevantes, en la figura 6 se muestra cuantos materiales entrego cada técnica, respecto a los OA relevantes de cada una.

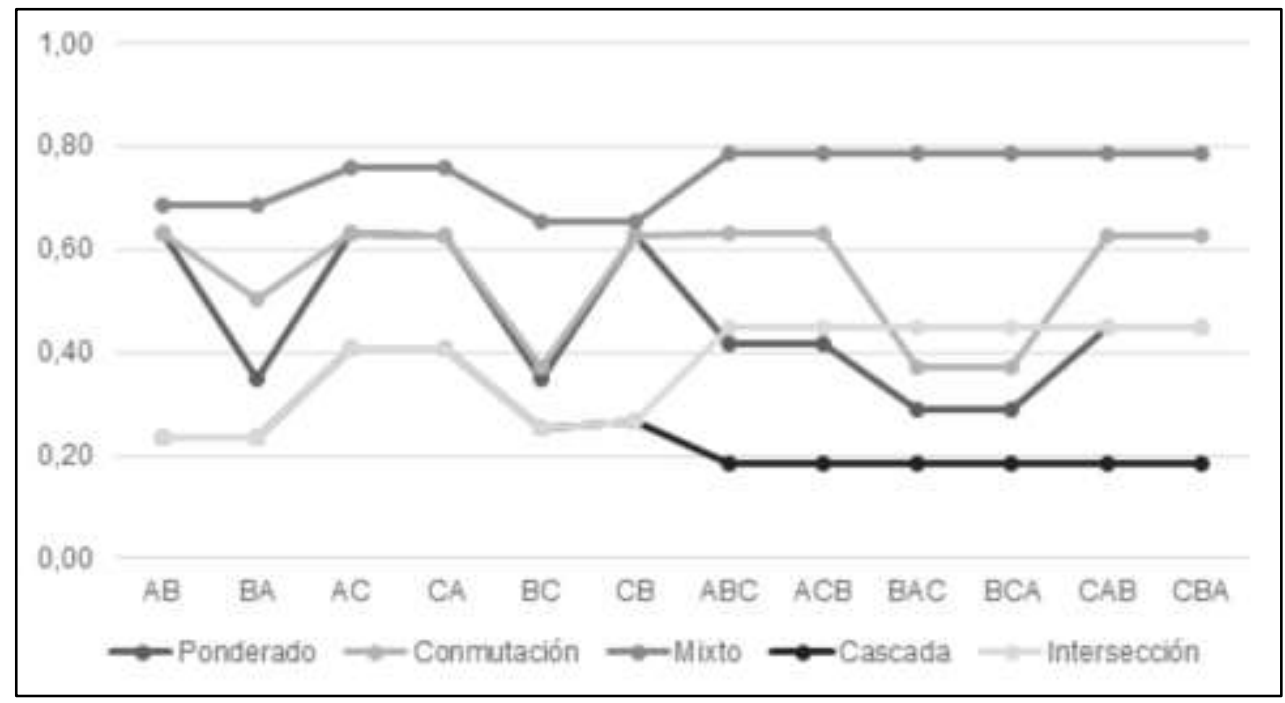

Fig. 5. Resultados de la métrica f1 score

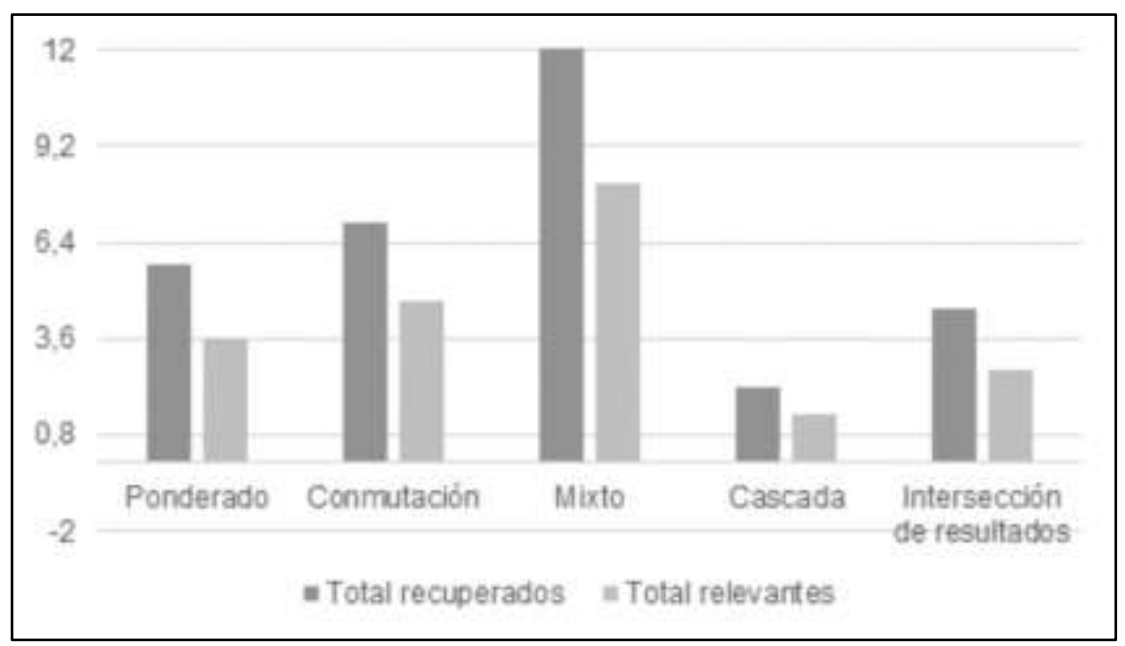

Fig. 6. Promedio de la cantidad de OA recuperados y relevantes para cada técnica de combinación

Con lo anterior se puede concluir que aunque la técnica de combinación mixta entrega mayor cantidad de OA relevantes, sin embargo, la cantidad de OA recuperados es mayor que en las otras técnicas. Por otro lado la técnica de cascada entrega muy pocos resultados. La combinación ideal sería aquella técnica que entregue una cantidad considerable de materiales de aprendizaje.

Para evaluar el desempeño general en la combinación de técnicas de recomendaciones (método híbrido) se consideran medidas estadísticas sobre los valores de precisión y recall. Dichas medidas se presentan de forma gráfica en los diagramas de caja (diagrama de bigotes) de la figura 7, agrupados que permiten hacer visualmente una comparación cuantitativa y cualitativa de los resultados. Los diagramas de caja ilustran medidas estadísticas así: en cada cuadro la marca central es la mediana, los bordes de la caja son los percentiles 25 y 75, los bigotes se extienden a los puntos de datos más extremos que no se consideran valores atípicos, y los valores atípicos se representan individualmente. 




Fig. 7. Diagrama de caja para los resultados de las métricas de precisión y de recall

Del diagrama de cajas se puede concluir que las técnicas híbridas de conmutación y mixto pueden entregar más cantidad de material educativo relevante, independientemente de las combinaciones de los sistemas de recomendación que se apliquen.

\section{CONCLUSIONES}

Utilizar sistemas de recomendación híbrida, mejora los resultados relevantes que se entregan a un usuario según sus características personales y adaptándose a sus necesidades. La principal ventaja de los sistemas híbridos es que ayuda a mejorar los problemas de inicialización y dependiendo de la técnica de combinación que se aplique los resultados son diferentes y la información con la que se cuenta. En este artículo se propone un método para desarrollar sistemas de recomendación híbrida basado en el perfil del estudiante con el fin de obtener resultados personalizados y adaptables, que apoyen su proceso de aprendizaje. Este método cumple con una serie de pasos que inician con la selección de características del perfil del estudiante, continúa con la selección de los algoritmos de recomendación, seguido de la elección en la combinación de los resultados de esas técnicas y finalmente se llega a la aplicación de métricas que evalúen las combinaciones. El aporte principal de este artículo está en la realización de pruebas de las diferentes técnicas de combinación de los resultados de los sistemas de recomendación (Contenido, colaborativo y conocimiento) y se evalúa las recuperaciones con las diferentes integraciones aplicando las medidas de precisión, recall y f1 score. Con las evaluaciones realizadas se puede concluir que la mejor combinación de SR será la que tenga en cuenta la recomendación por conocimiento, además que el método mixto es mejor porque si entrega todos los elementos relevantes de un conjunto de objetos, su desventaja es que el resultado puede contener una mayor cantidad de OA y para el estudiante será más difícil visualizar los $O A$ entregados. Sin embargo consideramos que la intersección de resultados puede ser más útil al momento de entregar los resultados, debido que entrega una cantidad de OA relevantes considerable.

Como trabajo futuro se propone tener en cuenta un orden de los resultados entregados por cada técnica de recomendación para evaluar mejores desempeños y tener un valor de importancia para cada OA según la posición en la lista de resultados. Además implementar técnicas de recomendación del estado del arte y hacer las combinaciones presentadas en este método para hacer comparaciones. Respecto a la retroalimentación del estudiante, se pueden tener valoraciones comparativas y subjetivas sobre la combinación de técnicas apoyado por el SR híbrido que les gusta más.

\section{AGRADECIMIENTOS}

El trabajo de investigación presentado en este artículo fue financiado parcialmente por el proyecto de COLCIENCIAS de la Universidad Nacional de Colombia titulado: "RAIM: Implementación de un framework apoyado en tecnologías móviles y de realidad aumentada para entornos educativos ubicuos, adaptativos, accesibles e interactivos para todos", con código 1119-569-34172. Adicionalmente, este trabajo fue desarrollado con el apoyo de la beca de doctorado del "Programa Nacional de Formación de Investigadores - COLCIENCIAS" otorgada a Paula Andrea Rodríguez Marín. 


\section{REFERENCIAS}

Acevedo, C., J.L. Arciniegas, X. García y J. Perrinet, Proceso de Adaptación de una Aplicación de eaprendizaje a t-aprendizaje, Información Tecnológica, Vol. 21(6), 27-36 (2010)

Alonso, C., Gallego, D., y Honey, P., Los Estilos de Aprendizaje. Procedimientos de diagnostico y mejora. Bilbao (1997)

Baeza-Yates, R., y Ribeiro-Neto, B., Modern Information Retrieval: The Concepts and Technology Behind Search. (A. Wesley, Ed.)Addision Wesley (Vol. 9) (2011)

Burke, R., Hybrid Recommender Systems: Survey and Experiments. User Modeling and User-Adapted Interaction, 12(4), 331-370 (2002)

Burke, R., Hybrid web recommender systems. The Adaptive Web, 4321, 377-408 (2007)

Cardona, D.M. y Sánchez, J.M., Indicadores Básicos para Evaluar el Proceso de Aprendizaje en Estudiantes de Educación a Distancia en Ambiente e-learning, Formación Universitaria, Vol. 3(6), 15-32 (2010)

Cazella, S. C., Reategui, E. B., y Nunes, M. A., A Ciência da Opinião: Estado da arte em Sistemas de Recomendação. JAI: Jornada de Atualização Em Informática Da SBC. Rio de Janeiro, RJ: PUC Rio, 161$216(2010)$

Chesani, F., Recommmendation Systems. Corso Di Laurea in Ingegneria Informatica, 1-32 (2002)

D'Agostino, E., Casali, A., Corti, R., y Torres, A., Sistema de Apoyo al Aprendizaje Diagnóstico Utilizando Perfiles de Usuario: EndoDiag II. VIII Simposio Argentino de Informática y Salud, pp. 1-14 (2005)

Degemmis, M., Lops, P., y Semeraro, G., A content-collaborative recommender that exploits WordNet-based user profiles for neighborhood formation. User Modeling and User-Adapted Interaction, 217-255. doi:10.1007/s11257-006-9023-4 (2007)

González, H., Duque Méndez, N., y Ovalle Carranza, D., Técnicas inteligentes para la actualización dinámica del perfil del usuario en un sistema de educación virtual. In Tendencias en Ingeniería de Software e Inteligencia Artificial (2009)

Hdioud, F., Frikh, B., y Ouhbi, B., A comparison study of some algorithms in Recommender Systems. Information Science and Technology (CIST), Colloquium, 130-135 (2012)

Klašnja-Milićević, A., Vesin, B., Ivanović, M., y Budimac, Z., E-Learning personalization based on hybrid recommendation strategy and learning style identification. Computers \& Education, 56(3), 885-899. doi:10.1016/j.compedu.2010.11.001 (2011)

Learning Technology Standards Committee, IEEE Standard for Learning Object Metadata. Institute of Electrical and Electronics Engineers, New York (2002).

Li, J. Z., Quality, Evaluation and Recommendation for Learning Object. International Conference on Educational and Information Technology, (Iceit), 533-537 (2010).

Ovalle, D.A., O.M. Salazar y N.D. Duque, Modelo de Recomendación Personalizada en Cursos Virtuales basado en Computación Ubicua y Agentes Inteligentes, Revista de Información Tecnológica. Vol . 25(6), pp. 131-142 (2014)

Puello, P., Fernández, D., y Cabarcas, A., Herramienta para la Detección de Estilos de Aprendizaje en Estudiantes utilizando la Plataforma Moodle. Formación Universitaria, 7(4), 15-24. doi:10.4067/S0718$50062014000400003(2014)$

Rodríguez, P. A., Moreno, J., Duque, N. D., Ovalle, D., y Silveira, R., Un modelo para la composición semiautomática de contenido educativo desde repositorios abiertos de objetos de aprendizaje. Revista Electrónica de Investigación Educativa (REDIE)., 16(1) (2014)

Rodríguez-Marín, P. A., Duque-Méndez, N. D., y Ovalle, D. A., Modelo de recomendación adaptativa de objetos de aprendizaje en el marco de una federación de repositorios, apoyado en agentes inteligentes y perfiles de usuario - Tesis de Maestría. Universidad Nacional de Colombia Sede Medellín (2013) 
Salehi, M., Pourzaferani, M., y Razavi, S. A., Hybrid attribute-based recommender system for learning material using genetic algorithm and a multidimensional information model. Egyptian Informatics Journal. doi:10.1016/j.eij.2012.12.001 (2013)

Tabares, V., Duque, N., Rodríguez, P., Moreno, J., y Ovalle, D., FROAC: Una Iniciativa Colombiana para la Integración de Repositorios de Objetos. Campus Virtuales, IV(1), 108-117 (2015)

Tovar, L. C., Bohórquez, J. a, y Puello, P., Propuesta metodológica para la construcción de objetos virtuales de aprendizaje basados en realidad aumentada. Formación Universitaria, 7(2), 11-20. doi:10.4067/S0718$50062014000200003(2014)$

Vekariya, V., y Kulkarni, G. R., Hybrid recommender systems: Survey and experiments. In 2012 Second International Conference on Digital Information and Communication Technology and its Applications (DICTAP) (pp. 469-473). leee. doi:10.1109/DICTAP.2012.6215409 (2012)

Zapata, A., Menendez, V., Prieto, M., y Romero, C., A Hybrid Recommender Method for Learning Objects. Design and Evaluation of Digital Content for Education Proceedings Published by International Journal of Computer Applications, 1-7 (2011) 